\title{
A missing link between nonlinear MPC schemes with guaranteed stability
}

\author{
Moritz Schulze Darup and Mark Cannon
}

\begin{abstract}
We present an interesting link between two nonlinear MPC schemes with guaranteed stability. In particular, we show that terminal sets and costs designed for MPC with terminal constraints can be exploited to compute suitable prediction horizons for stabilizing MPC without terminal constraints.
\end{abstract}

\section{INTRODUCTION}

Model predictive control (MPC) is an optimization based method for the feedback control of dynamical systems. It is well-known for its ability to consider both performance demands and constraint satisfaction. While MPC allows for (nearly) optimal closed-loop behavior, stability of the controlled system is not intrinsically guaranteed. Stability can, however, be guaranteed using different techniques. Among a variety of methods designed for different types of systems (see [1] for an overview), we can basically distinguish two independent approaches. First, by including a so-called terminal set (and an associated terminal cost) in the optimization problem, the predicted trajectory can be explicitly forced into a neighborhood of the origin, where a stabilizing controller is known (see, e.g., [2], [3] or Sect. IIA). Second, by suitably choosing the prediction horizon, we can implicitly guarantee that the optimal predicted trajectory ends in a stabilizing neighborhood (see, e.g., [4]-[6] or Sect. II-B).

The two approaches have well-known differences. The first approach allows to stabilize a system using relatively short prediction horizons while the second approach usually requires longer horizons. Although this is an advantage of the first method, it has to be taken into account that the inclusion of a terminal set and cost modifies the optimization problem and the underlying performance measure. In fact, the second approach usually provides closer approximations of the infinite-horizon cost. Finally, the two methods rely on different assumptions. The first approach requires the knowledge of an appropriate terminal set and an associated terminal cost function (see Thm. 1 in Sect. II). The second approach assumes some relations between the infinite horizon cost and the stage cost are known (see Thm. 2 in Sect. II). While methods for the computation of terminal sets and costs for a huge class of systems exist (see, e.g., [7]-[10] or Sect. III), procedures for the systematic verification of the conditions (8) and (9) in Thm. 2 are lacking.

*This research was funded by Deutsche Forschungsgemeinschaft (SCHU 2094/1-1)

M. Schulze Darup and M. Cannon are with the Control Group, Department of Engineering Science, University of Oxford, Parks Road, Oxford OX1 3PJ, UK. E-mail: moritz.schulzedarup@rub. de.
In this paper we intend to show that, despite the discussed differences, the two MPC schemes offer an interesting link. In particular, we present a method that allows verification of the conditions in Thm. 2 (associated with the second method) based on the knowledge of a terminal set and cost as required for the first method. In other words, the systematic verification of the assumptions underlying both methods can be carried out using the same numerical methods.

To establish this result, we briefly recall two variants of nonlinear MPC schemes with guaranteed stability in Sect. II. We then introduce a universal condition for the identification of terminal sets and costs in Sect. III (see Prop. 1) and show that established criteria can be easily converted to this form. The main result of the paper, i.e., the systematic verification of conditions (8) and (9), is presented in Sect. IV. The link between the two discussed MPC schemes is illustrated in Sect. V based on a numerical example. Finally, conclusions are stated in Sect. VI.

\section{A. Notation}

We denote non-negative reals, positive reals and positive natural numbers by $\mathbb{R}_{0}, \mathbb{R}_{+}$and $\mathbb{N}_{+}$, respectively. In addition, we define $\mathbb{N}_{[i, k]}:=\{j \in \mathbb{N} \mid i \leq j \leq k\}$. Let $P \in \mathbb{R}^{n \times n}$ and $H \in \mathbb{R}^{t \times n}$. We write $P \succ 0$ if $P$ is positive definite and symmetric. Beside the vector norms $\|x\|_{1},\|x\|_{2}$, and $\|x\|_{\infty}$, we use $\|x\|_{P}=\sqrt{x^{T} P x}$ (for $P \succ 0$ ) and the matrix norm $\|H\|_{\infty}=\max _{i \in \mathbb{N}_{[1, t]}} \sum_{j=1}^{n}\left|H_{i j}\right|$. Whenever we consider $e_{k}^{T} H$ or $H e_{j}, e_{k} \in \mathbb{R}^{t}$ and $e_{j} \in \mathbb{R}^{n}$ refer to the $k$-th and the $j$-th Euclidean unit vector, respectively. Let $\mathcal{C} \subset \mathbb{R}^{n}$ be a compact set. By $\partial \mathcal{C}$ and $\operatorname{int}(\mathcal{C})$, we denote the boundary and the interior and of $\mathcal{C}$. For a polytope $\mathcal{C}, \operatorname{extr}(\mathcal{C})$ denotes the set of all vertices (or extreme points) of $\mathcal{C}$. Conversely, $\operatorname{conv}\left(\left\{v_{1}, \ldots, v_{s}\right\}\right)$ refers to the convex hull of the points $v_{1}$ through $v_{s}$. We call a set $\mathcal{C}$ a $\mathrm{C}$-set if $\mathcal{C}$ is compact, convex and $0 \in \operatorname{int}(\mathcal{C})$. Scaling of $\mathcal{C}$ by a factor $\rho>0$ is understood as $\rho \mathcal{C}=\{\rho x \mid x \in \mathcal{C}\}$. Finally, for a $\mathrm{C}$-set $\mathcal{C}$, the Minkowski function is defined as $\Psi_{\mathcal{C}}(x)=\inf \left\{\lambda \in \mathbb{R}_{+} \mid x \in \lambda \mathcal{C}\right\}$.

\section{REVIEW OF STABILIZING MPC SCHEMES}

Consider the nonlinear discrete-time system

$$
x(k+1)=f(x(k), u(k)), \quad x(0)=x_{0}
$$

with state and input constraints of the form

$$
x(k) \in \mathcal{X} \quad \text { and } \quad u(k) \in \mathcal{U} \quad \text { for every } \quad k \in \mathbb{N} .
$$

The control task is to steer the system to the origin while minimizing the infinite horizon cost in (1). Taking the system 
dynamics and the constraints into account, this task can be formulated as the optimal control problem

$$
\begin{aligned}
& V_{\infty}\left(x_{0}\right):=\min _{\mathbf{u}_{\infty}} \sum_{k=0}^{\infty} l(x(k), u(k)) \\
& \text { s.t. } x(0)=x_{0} \text {, } \\
& x(k+1)=f(x(k), u(k)), \quad \forall k \in \mathbb{N}, \\
& x(k) \in \mathcal{X} \text {, } \\
& u(k) \in \mathcal{U} \text {, } \\
& \forall k \in \mathbb{N} \text {, } \\
& \forall k \in \mathbb{N} \text {, }
\end{aligned}
$$

where $l: \mathbb{R}^{n} \times \mathbb{R}^{m} \rightarrow \mathbb{R}_{0}$ is the stage cost and where $\mathbf{u}_{\infty}=$ $\{u(0), u(1), \ldots\}$ is the infinite sequence of control actions to be chosen. A meaningful cost function is obtained under the following assumptions that we consider throughout the paper.

Assumption 1: The origin is an equilibrium point of the system, i.e., $f(0,0)=0$. The constraints $\mathcal{X} \subset \mathbb{R}^{n}$ and $\mathcal{U} \subset$ $\mathbb{R}^{m}$ are C-sets. The stage cost $l(x, u)$ is convex and positive definite (in $x$ and $u$ ).

Problem (1) is generally hard to solve since it is infinite dimensional and non-convex. In MPC, (1) is thus replaced by the truncated optimal control problem

$$
\begin{aligned}
& V_{N}\left(x_{0}\right):=\min _{\mathbf{u}_{N}} \tau(x(N))+\sum_{k=0}^{N-1} l(x(k), u(k)) \\
& \text { s.t. } \quad x(0)=x_{0} \text {, } \\
& x(k+1)=f(x(k), u(k)), \quad \forall k \in \mathbb{N}_{[0, N-1]}, \\
& x(k) \in \mathcal{X} \text {, } \\
& u(k) \in \mathcal{U} \text {, } \\
& x(N) \in \mathcal{T} \text {, }
\end{aligned}
$$

with prediction horizon $N \in \mathbb{N}_{+}$, terminal cost $\tau: \mathbb{R}^{n} \rightarrow$ $\mathbb{R}_{0}$ and terminal set $\mathcal{T} \subseteq \mathcal{X}$. While (2) may still be nonconvex, the number of decision variables (in terms of the input sequence $\mathbf{u}_{N}=\{u(0), \ldots, u(N-1)\}$ ) is finite. Let $\mathbf{u}_{N}^{*}$ be an optimizer of (2) at $x_{0}$. Then, the MPC feedback law $\varrho_{N}: \mathbb{R}^{n} \rightarrow \mathbb{R}^{m}$ at $x_{0}$ is defined by

$$
\varrho_{N}\left(x_{0}\right):=u^{*}(0),
$$

where $u^{*}(0)$ is the first element of the optimal input sequence $\mathbf{u}_{N}^{*}$. Depending on the choice of $\tau, \mathcal{T}$, and $N$ the origin of the controlled system

$$
x(k+1)=f\left(x(k), \varrho_{N}(x(k))\right.
$$

may or may not be asymptotically stable. We recall two suitable choices guaranteeing stability in the following.

\section{A. Stabilizing MPC with terminal constraints}

According to [1, Assum. A1 to A4], [2], and [3], a stabilizing MPC scheme is obtained by choosing the terminal $\operatorname{cost} \tau$ and the terminal set $\mathcal{T}$ as summarized in Thm. 1. We refer to this approach as MPC with terminal constraints since we usually have $\mathcal{T} \subset \mathcal{X}$ and thus further restrict the terminal state $x(N)$.
Theorem 1: Assume there exists a set $\mathcal{C} \subseteq \mathcal{X}$ with $0 \in$ $\operatorname{int}(\mathcal{C})$ and a function $\varphi: \mathbb{R}^{n} \rightarrow \mathbb{R}_{0}$ such that, for every $x \in \mathcal{C}$, there exists a $u \in \mathcal{U}$ such that $f(x, u) \in \mathcal{C}$ and

$$
\varphi(f(x, u)) \leq \varphi(x)-l(x, u) .
$$

Set $\tau(\cdot)=\varphi(\cdot)$ and $\mathcal{T}=\mathcal{C}$ in (2). Then, for every choice $N \geq 1$, the origin of the controlled system (4) is asymptotically stable with domain of attraction (DOA) equal to $\mathcal{D}_{N}=\left\{x_{0} \in \mathcal{X} \mid(2)\right.$ is feasible $\}$.

It is important to note that (5) implies

$$
V_{\infty}(x) \leq \varphi(x) \text { for every } \quad x \in \mathcal{C},
$$

i.e., $\varphi$ represents an overapproximation of the infinite horizon cost (1) on $\mathcal{C}$.

\section{B. Stabilizing MPC without terminal constraints}

According to [4, Thm. 4 and Rem. 5], a stabilizing MPC scheme can also be obtained by choosing the prediction horizon $N$ according to the criteria in Thm. 2. As a preparation, we introduce the function $l^{\star}: \mathbb{R}^{n} \rightarrow \mathbb{R}$ with

$$
l^{\star}(x):=\inf _{u \in \mathcal{U}} l(x, u)
$$

Theorem 2: Assume there exists a set $\mathcal{C} \subseteq \mathcal{X}$ with $0 \in$ $\operatorname{int}(\mathcal{C})$ and constants $\gamma, \mu \in \mathbb{R}_{+}$such that,

$$
\begin{aligned}
V_{\infty}(x) \leq \gamma l^{\star}(x) & \text { for every } \quad x \in \mathcal{C}, \\
\mu \leq l^{\star}(x) & \text { for every } \quad x \in \mathcal{X} \backslash \operatorname{int}(\mathcal{C}),
\end{aligned}
$$

and, for every $x \in \mathcal{C}$, there exists a $u \in \mathcal{U}$ such that $f(x, u) \in$ $\mathcal{C}$. Set $\tau(\cdot)=l^{\star}(\cdot)$ and $\mathcal{T}=\mathcal{X}$. Then, for every choice $N \in \mathbb{N}_{+}$and $\delta \in \mathbb{R}_{+}$such that

$$
\frac{\ln (\beta)}{\ln (\beta)-\ln (\beta-1)}<N,
$$

where $\beta=\max \{\delta / \mu, \gamma, 2\}$, the origin of the controlled system is asymptotically stable with DOA larger than or equal to $\mathcal{D}_{N}=\left\{x_{0} \in \mathcal{X} \mid V_{N}\left(x_{0}\right) \leq \delta\right\}$.

Remark 1: Condition (10) slightly differs from the original conditions stated in [4]. In fact, the conditions from [4, Thm. 4 and Rem. 5] read

$$
\delta\left(\frac{\beta-1}{\beta}\right)^{\widehat{N}-1}<\mu \text { and } \frac{\ln (\beta-1)}{\ln (\beta)-\ln (\beta-1)}+2<\widehat{N} .
$$

To see that conditions (11) and (10) are interchangeable, first note that, due to $\beta \geq \frac{\delta}{\mu}$, the first condition in (11) holds whenever the second condition in (11) is satisfied. Moreover, (2) with $\tau(x)=l^{\star}(x)$ and horizon length $N$ (as considered in Thm. 2) is equal to (2) with $\tau(x)=0$ (as considered in [4, Sect. 2]) and horizon length $N+1$. Clearly, the second relation in (11) holds for $\widehat{N}=N+1$ if and only if (10) holds for $N$.

Since Thm. 2 does not (further) restrict the terminal state $x(N)$ (since $\mathcal{T}=\mathcal{X}$ ), we refer to this approach as $M P C$ without terminal constraints. Note that the choice $\tau(x)=$ $l^{\star}(x)$ requires $l^{\star}(x)$ to be known explicitly. If this is not the case, we can instead use $\tau(x)=0$ and $N=\widehat{N}$ with $\widehat{N}$ satisfying (11). 


\section{IDENTIFICATION OF TERMINAL SETS AND COSTS}

As mentioned in the introduction, there exist a number of methods that can (in principle) be used to compute terminal sets $\mathcal{C}$ and terminal costs $\varphi$ as required in Thm. 1 (see, e.g., [7]-[10]). In many cases, the resulting set $\mathcal{C}$ and function $\varphi$ can be characterized using the concepts introduced in Prop. 1. The universal characterization of $\mathcal{C}$ and $\varphi$ based on the Minkowski function $\Psi_{\mathcal{C}}$ will be instrumental for the systematic verification of the conditions (8) and (9) addressed in Sect. IV.

Proposition 1: Let $\mathcal{C} \subseteq \mathcal{X}$ be a C-set, $\eta \in \mathbb{R}_{+}$, and $\kappa \in$ $\mathbb{N}_{+}$. Assume, for every $x \in \mathcal{C}$, there exists a $u \in \mathcal{U}$ such that

$$
\Psi_{\mathcal{C}}^{\kappa}(f(x, u)) \leq \Psi_{\mathcal{C}}^{\kappa}(x)-\eta l(x, u) .
$$

Then $\mathcal{C}$ and $\varphi(x)=\frac{1}{\eta} \Psi_{\mathcal{C}}^{\kappa}(x)$ are as required in Thm. 1 .

Proof: Let $x \in \mathcal{C}$ be arbitrary but fixed. Assume $u \in \mathcal{U}$ is such that (12) holds. Dividing (12) by $\eta>0$ and substituting $\frac{1}{\eta} \Psi_{\mathcal{C}}^{\kappa}(x)$ by $\varphi(x)$ immediately leads to (5). It remains to prove $f(x, u) \in \mathcal{C}$. Since $l(x, u) \geq 0$ and $\eta>0$, (5) implies $\Psi_{\mathcal{C}}(f(x, u)) \leq \Psi_{\mathcal{C}}(x)$.

In principal, the reformulation of the criteria in [7]-[10] in the style of condition (12) is straighforward. However, in [7], continuous-time systems are considered. In [9], [10], controlled invariant sets are computed, which implies $\eta=0$ in (12). Clearly, for $\eta=0$, the function $\varphi(x)=\frac{1}{\eta} \Psi_{\mathcal{C}}^{\kappa}(x)$ is ill-defined. Finally, in [8], the stage cost $l(x, u) \stackrel{\eta}{=}\|Q x\|_{\infty}$ is addressed, which does not satisfy Assum. 1 (since $l(x, u)$ is not pos. def. in $u$ ). Thus, in order to prepare the results in Sect. IV, we adapt two criteria introduced in [7] and [8] in the following and rewrite them according to Prop. 1. The adaptation of the criteria in [9] and [10] can be carried out analogously but is omitted here due to space limitations.

\section{A. Criteria for terminal ellipsoids and polytopes}

Analogously to [7], [8], we assume the nonlinear system can be bounded using linear difference inclusions (LDIs). In particular, let there be matrices $A_{1}, \ldots, A_{r} \in \mathbb{R}^{n \times n}$ and $B_{1}, \ldots, B_{r} \in \mathbb{R}^{n \times m}, r \in \mathbb{N}_{+}$, such that

$$
\begin{gathered}
f(x, u) \in \operatorname{conv}\left(\left\{A_{1} x+B_{1} u, \ldots, A_{r} x+B_{r} u\right\}\right) \\
\text { for every } x \in \mathcal{C} \text { and every } u \in \mathcal{U} .
\end{gathered}
$$

Then an ellipsoidal terminal set can be identified as follows.

Corollary 1: Let $\eta \in \mathbb{R}_{+}, P \in \mathbb{R}^{n \times n}$, and $K \in \mathbb{R}^{m \times n}$ with $P \succ 0$. Consider the quadratic stage cost

$$
l(x, u)=\|x\|_{Q}^{2}+\|u\|_{R}^{2} .
$$

with $Q \succ 0$ and $R \succ 0$. Assume the ellipsoidal set

$$
\mathcal{C}=\left\{x \in \mathbb{R}^{n} \mid\|x\|_{P}^{2} \leq 1\right\}
$$

is such that $\mathcal{C} \subseteq\{x \in \mathcal{X} \mid K x \in \mathcal{U}\}$ and assume (13) holds for every $x \in \mathcal{C}$ and every $u \in \mathcal{U}$. If

$$
\left(A_{i}+B_{i} K\right)^{T} P\left(A_{i}+B_{i} K\right)-P+\eta\left(Q+K^{T} R K\right) \preceq 0
$$

for every $i \in \mathbb{N}_{[1, r]}$, then $\mathcal{C}$ and $\varphi(x)=\frac{1}{\eta} \Psi_{\mathcal{C}}^{2}(x)$ are as required in Thm. 1 .

Proof: Since $\Psi_{\mathcal{C}}^{2}(x)=\|x\|_{P}^{2}$, (16) implies

$$
\Psi_{\mathcal{C}}^{2}\left(\left(A_{i}+B_{i} K\right) x\right) \leq \Psi_{\mathcal{C}}^{2}(x)-\eta l(x, K x)
$$

for every $x \in \mathbb{R}^{n}$. Moreover, (13) and $K x \in \mathcal{U}$ yields

$$
f(x, K x) \in \operatorname{conv}\left(\left\{\left(A_{1}+B_{1} K\right) x, \ldots,\left(A_{r}+B_{r} K\right) x\right\}\right)
$$

for every $x \in \mathcal{C}$. Convexity of the set $\mathcal{C}$ and the functions $\Psi_{\mathcal{C}}$ and $l$ immediately leads to (12).

The criterion in Cor. 1 is linked to quadratic stage costs. For an arbitrary cost function $l$ (satisfying Assum. 1), a polytopic terminal set can be identified according to Cor. 2 .

Corollary 2: Let $\eta \in \mathbb{R}_{+}$and let $H \in \mathbb{R}^{t \times n}$ be such that the polytopic set

$$
\mathcal{C}:=\left\{x \in \mathbb{R}^{n} \mid H x \leq \mathbf{1}\right\},
$$

where $1 \in \mathbb{R}^{t}$ is a vector with all entries equal to 1 , is a C-set with $\mathcal{C} \subseteq \mathcal{X}$. Let $\left\{v_{1}, \ldots, v_{s}\right\}=\operatorname{extr}(\mathcal{C})$ and assume (13) holds. If, for every $v_{j}$, there exists a $w_{j} \in \mathcal{U}$ such that

$$
e_{k}^{T} H\left(A_{i} v_{j}+B_{i} w_{j}\right) \leq 1-\eta l\left(v_{j}, w_{j}\right)
$$

for every $i \in \mathbb{N}_{[1, s]}$ and every $k \in \mathbb{N}_{[1, t]}$, then $\mathcal{C}$ and $\varphi(x)=$ $\frac{1}{\eta} \Psi_{\mathcal{C}}(x)$ are as required in Thm. 1.

Proof: Relation (19) implies

$$
\Psi_{\mathcal{C}}\left(A_{i} v_{j}+B_{i} w_{j}\right) \leq \Psi_{\mathcal{C}}\left(v_{j}\right)-\eta l\left(v_{j}, w_{j}\right)
$$

for every $i \in \mathbb{N}_{[1, s]}$ and every $k \in \mathbb{N}_{[1, t]}$. Using convexity of the sets $\mathcal{C}$ and $\mathcal{U}$ and the functions $\Psi_{\mathcal{C}}$ and $l$ as well as positive homogeneity of $\Psi_{\mathcal{C}}$ with degree 1 (see $[11$, p. 80]) allows the proof of (12).

\section{CHOICE OF PREDICTION HORIZONS}

The terminal sets $\mathcal{C}$ and costs $\varphi$ identified according to Prop. 1 (resp. Cors. 1 and 2) are instrumental to design stabilizing MPC schemes with terminal constraints according to Thm. 1. In this section we show that $\mathcal{C}$ and $\varphi$ as in Prop. 1, can also be exploited to compute $\gamma$ and $\mu$ satisfying the conditions (8) and (9) in Thm. 2. In combination with the user-defined choice of $\delta$, the constants $\gamma$ and $\mu$ define $\beta$ and consequently allow the choice of an appropriate prediction horizon $N$ according to (10). Thus, $\mathcal{C}$ and $\varphi$ as in Prop. 1 can also be used to design stabilizing MPC schemes without terminal constraints according to Thm. 2. The relation between $\mathcal{C}$ and $\varphi$ as in Prop. 1 and $\gamma$ and $\mu$ satisfying (8) and (9) is summarized in Prop. 2 further below. As a preparation, we prove the following lemma.

Lemma 1: Let $\eta, \kappa$, and $\mathcal{C}$ be as in Prop. 1 and assume (12) holds. Let $\rho \in \mathbb{R}_{+}$, assume

$$
l^{\star}(x)=\Psi_{\mathcal{L}}^{\kappa}(x) \text { for every } \quad x \in \mathcal{X},
$$

and define

$$
\mathcal{L}:=\left\{x \in \mathbb{R}^{n} \mid l^{\star}(x) \leq 1\right\}
$$

Then

$$
\mathcal{L} \subseteq \rho \mathcal{C}
$$

is necessary and sufficient for the conditions

$$
\begin{array}{ccrl}
\varphi(x) \leq \frac{\rho^{k}}{\eta} l^{\star}(x) & \text { for every } & x \in \mathcal{C}, \quad \text { and } \\
\frac{1}{\rho^{k}} \leq l^{\star}(x) & \text { for every } & x \in \mathcal{X} \backslash \operatorname{int}(\mathcal{C}),
\end{array}
$$

to hold. 
Proof: Necessity: Assume (22) does not hold. Then, since $\mathcal{C}$ and $\mathcal{L}$ are $\mathrm{C}$-sets by assumption and construction, respectively, there exists an $x \in \partial \mathcal{C}$ such that $\rho x \in \operatorname{int}(\mathcal{L})$. Let $\epsilon=\Psi_{\mathcal{L}}^{\kappa}(\rho x)$ and note that $\epsilon \in(0,1)$. Thus,

$$
\varphi(x)=\frac{1}{\eta} \Psi_{\mathcal{C}}^{\kappa}(x)=\frac{1}{\eta}>\frac{\epsilon}{\eta}=\frac{\rho^{k}}{\eta} \Psi_{\mathcal{L}}^{\kappa}(x)=\frac{\rho^{k}}{\eta} l^{\star}(x),
$$

which contradicts (23). Moreover, $1>\epsilon=\rho^{\kappa} l^{*}(x)$ contradicts (24).

Sufficiency: From (22) we infer $\Psi_{\mathcal{L}}(x) \geq \Psi_{\rho \mathcal{C}}(x)=$ $\frac{1}{\rho} \Psi_{\mathcal{C}}(x)$ which implies $\Psi_{\mathcal{C}}^{\kappa}(x) \leq \rho^{\kappa} \Psi_{\mathcal{L}}^{\kappa}(x)$. Since we have $\varphi(x)=\frac{1}{\eta} \Psi_{\mathcal{C}}^{\kappa}(x)$ according to Prop. 1, we find

$$
\varphi(x)=\frac{1}{\eta} \Psi_{\mathcal{C}}^{\kappa}(x) \leq \frac{\rho^{\kappa}}{\eta} \Psi_{\mathcal{L}}^{\kappa}(x)=\frac{\rho^{\kappa}}{\eta} l^{\star}(x),
$$

which proves (23).

We prove (24) by contradiction. To this end, assume (24) does not hold. Then, there exists an $x \in \mathcal{X} \backslash \operatorname{int}(\mathcal{C})$ such that $l^{\star}(x)<\frac{1}{\rho^{\kappa}}$. Thus, $\Psi_{\mathcal{C}}^{\kappa}(x) \geq 1$ and $1>\rho^{\kappa} \Psi_{\mathcal{L}}^{\kappa}(x)$, which implies $\Psi_{\mathcal{C}}^{\kappa}(x)>\rho^{\kappa} \Psi_{\mathcal{L}}^{\kappa}(x)$. This, however, contradicts (22) since (22) implies $\Psi_{\mathcal{C}}^{\kappa}(x) \leq \rho^{\kappa} \Psi_{\mathcal{L}}^{\kappa}(x)$.

Proposition 2: Let $\eta, \kappa$, and $\mathcal{C}$ be as in Prop. 1 and let $\rho$ and $\mathcal{L}$ be as in Lem. 1. Assume (12), (20), and (22) hold. Then $\mathcal{C}, \gamma=\frac{\rho^{\kappa}}{\eta}$, and $\mu=\frac{1}{\rho^{\kappa}}$ are as required in Thm. 2 .

Proof: We have $V_{\infty}(x) \leq \varphi(x)$ for every $x \in \mathcal{C}$ according to (6). Thus, $\mathcal{C}, \gamma=\frac{\rho^{\kappa}}{\eta}$, and $\mu=\frac{1}{\rho^{\kappa}}$ satisfy the conditions (8) and (9) according to Lem. 1.

Remark 2: Obviously, the statement in Lem. 1 is more strict than Prop. 2. To see this, first note that the conditions (8) and (23) as well as (9) and (24) are related to each other. As stated in Lem. 1, condition (22) is necessary and sufficient for (23) and (24) to hold. Using the same argumentation as in the proof of Lem. 1, it is straightforward to show that (22) is also necessary and sufficient for (9) to hold with $\mu=\frac{1}{\rho^{\kappa}}$. However, it is important to note that (22) is only sufficient for (8) to hold with $\gamma=\frac{\rho^{\kappa}}{\eta}$. In fact, due to the overestimation (6), relation (8) may hold for $\gamma=\frac{\rho^{\kappa}}{\eta}$ even though (22) does not.

The computation of the constants $\gamma$ and $\mu$ according to Prop. 2 basically requires the identification of a suitable $\rho$ satisfying the geometrical condition (22). However, Prop. 2 can only be applied if (20) holds. Condition (20) seems quite restrictive at first sight. We provide a detailed discussion of this condition in the remainder of this section. To this end, we address different combinations of terminal sets $\mathcal{C}$ and stage costs $l$ and discuss the applicability of Prop. 2. In particular, we consider ellipsoidal or polytopic sets $\mathcal{C}$ and quadratic or (piecewise) linear stage costs $l$, where the latter is defined as

$$
l(x, u)=\|Q x\|_{p}+\|R u\|_{p}
$$

with full-rank matrices $Q \in \mathbb{R}^{n \times n}$ and $R \in \mathbb{R}^{m \times m}$ and $p \in\{1, \infty\}$. Taking into account that ellipsoidal terminal sets can only be identified for quadratic stage costs (see Cor. 1), we obtain the four combinations listed in Tab. I. Anticipating the detailed analysis in Sects. IV-A through IV-D, Prop. 2 is applicable for the three combinations marked with " $\checkmark$ " in
Tab. I. Condition (20) is violated, however, for polytopic sets $\mathcal{C}$ in combination with quadratic stage costs $l$.

TABLE I

Applicability of Prop. 2 for different sets $\mathcal{C}$ and stage costs $l$.

\begin{tabular}{cccc}
\hline set $\mathcal{C}$ & \multicolumn{3}{c}{ stage cost $l$} \\
\cline { 2 - 4 } & quadratic & linear, $p=1$ & linear, $p=\infty$ \\
\hline ellipsoidal & $\checkmark /$ IV-A & - & - \\
polytopic & $\downarrow /$ IV-D & $\checkmark /$ IV-B & $\checkmark /$ IV-C \\
\hline
\end{tabular}

Sections IV-A through IV-D address the specification of Prop. 2 w.r.t. the four combinations of $\mathcal{C}$ and $l$ itemized in Tab. I. Special emphasis is placed on the reformulation of condition (22). In fact, according to Rem. 3, it is desirable to provide conditions that allow to easily compute the smallest $\rho$ satisfying (22).

Remark 3: According to (10), for smaller values of $\beta, N$ can be chosen to be smaller. A small $\beta$ is achieved for small $\gamma$ and large $\mu$. Regarding Prop. 2, both are obtained for a small choice of $\rho$.

\section{A. Ellipsoidal set and quadratic cost}

Corollary 3: Consider the quadratic cost (14). Let $\eta, \rho \in$ $\mathbb{R}_{+}$and $P \in \mathbb{R}^{n \times n}$ with $P \succ 0$. Assume the ellipsoidal set $\mathcal{C}$ as in (15) satisfies the conditions in Lem. 1 for $\kappa=2$ and

$$
\rho^{2} Q \succeq P
$$

Then $\mathcal{C}, \gamma=\frac{\rho^{2}}{\eta}$, and $\mu=\frac{1}{\rho^{2}}$ are as required in Thm. 2 .

Proof: First note that $l^{\star}(x)=\|x\|_{Q}^{2}=\Psi_{\mathcal{L}}^{2}(x)$ for every $x \in \mathcal{X}$, where $\mathcal{L}$ is defined as in (21). Thus, the claim immediately follows from Prop. 2 since (26) implies (22). To see this, note that (26) implies $\rho^{2}\|x\|_{Q}^{2} \geq\|x\|_{P}^{2}$. Hence, $x \in \mathcal{L}$, i.e., $\|x\|_{Q}^{2} \leq 1$, yields $\|x\|_{P}^{2} \leq \rho^{2}$, i.e., $x \in \rho \mathcal{C}$.

The smallest $\rho$ satisfying (26) can be easily found by solving a semi-definite program (SDP).

\section{B. Polytopic set and linear cost $(p=1)$}

Corollary 4: Consider the linear cost (25) with $p=1$. Let $\eta, \rho \in \mathbb{R}_{+}$and $H \in \mathbb{R}^{t \times n}$. Assume the polytopic set $\mathcal{C}$ as in (18) satisfies the conditions in Lem. 1 for $\kappa=1$ and

$$
\left(\begin{array}{c} 
\pm H Q^{-1} e_{1} \\
\vdots \\
\pm H Q^{-1} e_{n}
\end{array}\right) \leq \rho \mathbf{1}
$$

Then $\mathcal{C}, \gamma=\frac{\rho}{\eta}$, and $\mu=\frac{1}{\rho}$ are as required in Thm. 2 .

Proof: We have $l^{\star}(x)=\|Q x\|_{1}=\Psi_{\mathcal{L}}(x)$ for every $x \in \mathcal{X}$, with $\mathcal{L}$ as in (21). It remains to show (27) implies (22). To this end, note that (22) here reads $\left\{x \mid\|Q x\|_{1} \leq\right.$ $1\} \subseteq\{x \mid H x \leq \rho \mathbf{1}\}$. Using the coordinate transformation $x=Q^{-1} \xi$, this is equivalent to $\widehat{\mathcal{L}}=\left\{\xi \mid\|\xi\|_{1} \leq 1\right\} \subseteq$ $\left\{\xi \mid H Q^{-1} \xi \leq \rho \mathbf{1}\right\}=\widehat{\mathcal{C}}$. Since (the cross-polytope) $\widehat{\mathcal{L}}$ is convex, (22) holds if the vertices $\pm e_{1}, \ldots, \pm e_{n}$ of $\widehat{\mathcal{L}}$ are contained in $\widehat{\mathcal{C}}$, i.e., if (27) holds.

The smallest $\rho$ satisfying (27) can in principle be found by solving a linear program (LP). The numerical solution of 
the optimization problem is however not necessary, since it is easy to show that the solution reads

$$
\rho^{*}=\max _{j \in \mathbb{N}_{[1, n]}} \max _{k \in \mathbb{N}_{[1, t]}}\left|e_{k}^{T} H Q^{-1} e_{j}\right|
$$

\section{Polytopic set and linear cost $(p=\infty)$}

Corollary 5: Consider the linear cost (25) with $p=\infty$. Let $\eta \in \mathbb{R}_{+}$and $H \in \mathbb{R}^{t \times n}$. Assume the polytopic set $\mathcal{C}$ as in (18) satisfies the conditions in Lem. 1 for $\kappa=1$ and

$$
\left(\begin{array}{c}
H Q^{-1} \varepsilon_{1} \\
\vdots \\
H Q^{-1} \varepsilon_{2^{n}}
\end{array}\right) \leq \rho \mathbf{1},
$$

where $\epsilon_{1}, \ldots, \epsilon_{2^{n}} \in \mathbb{R}^{n}$ denote the $2^{n}$ vertices of the $\infty$ norm unit ball $\left\{\xi \in \mathbb{R}^{n} \mid\|\xi\|_{\infty} \leq 1\right\}$. Then $\mathcal{C}, \gamma=\frac{\rho}{\eta}$, and $\mu=\frac{1}{\rho}$ are as required in Thm. 2 .

Proof: The proof is analogous to the proof of Cor. 4. We obtain the condition $\widehat{\mathcal{L}}=\left\{\xi \mid\|\xi\|_{\infty} \leq 1\right\} \subseteq\left\{\xi \mid H Q^{-1} \xi \leq\right.$ $\rho \mathbf{1}\}=\widehat{\mathcal{C}}$, which is equivalent to $(28)$.

The smallest $\rho$ satisfying (27) reads $\rho^{*}=\left\|H Q^{-1}\right\|_{\infty}$.

\section{Polytopic set and quadratic cost}

In case of a polytopic terminal set $\mathcal{C}$, we have $\varphi(x)=$ $\frac{1}{\eta} \Psi_{\mathcal{C}}(x)$ according to Cor. 2 . In other words, (12) holds with $\kappa=1$. Unfortunately, we find

$$
l^{\star}(x)=\|x\|_{Q}^{2}=\Psi_{\mathcal{L}}^{2}(x) \quad \text { but } \quad l^{\star}(x) \neq \Psi_{\mathcal{L}}(x)
$$

for a quadratic stage cost $l$. Consequently, condition (20) does not hold and Prop. 2 cannot be applied. Nevertheless, a polytopic terminal set $\mathcal{C}$ can be used to compute $\gamma$ and $\mu$ even though a quadratic cost function is considered. In this case, however, the knowledge of an auxiliary ellipsoidal terminal set $\mathcal{E}$ is required. The constructive combination of the sets $\mathcal{C}$ and $\mathcal{E}$ is described in the following proposition. Conditions under which Prop. 3 is useful (resp. not useful) are discussed in Rem. 4 further below.

Proposition 3: Consider the quadratic cost (14). Let $\eta, \rho_{1}, \rho_{2} \in \mathbb{R}_{+}, H \in \mathbb{R}^{t \times n}$, and $P \in \mathbb{R}^{n \times n}$ with $P \succ 0$. Assume the polytopic set $\mathcal{C}$ as in (18) satisfies the conditions in Lem. 1 for $\kappa=1$ and

$$
\rho_{1} \geq \max _{k \in \mathbb{N}_{[1, t]}}\left\|e_{k}^{T} H L^{-T}\right\|_{2},
$$

where $L$ refers to the Cholesky decomposition of $Q$, i.e., $L L^{T}=Q$. In addition, assume the set $\mathcal{E}=\left\{x \mid x^{T} P x \leq 1\right\}$ satisfies the conditions ${ }^{1}$ in Lem. 1 for $\kappa=2$ and

$$
\rho_{2}^{2} Q \succeq P \text {. }
$$

Then, $\mathcal{C}, \gamma=\frac{\max \left\{\rho_{1} \rho_{2}, \rho_{2}^{2}\right\}}{\eta}$, and $\mu=\frac{1}{\rho_{1}^{2}}$ are as required in Thm. 2.

Proof: We first show that (29) and (30) imply

$$
\mathcal{L} \subseteq \rho_{1} \mathcal{C} \quad \text { and } \quad \mathcal{L} \subseteq \rho_{2} \mathcal{E} .
$$

The second relation in (31) follows with the same arguments as in the proof of Cor. 3. It remains to prove $\mathcal{L} \subseteq \rho_{1} \mathcal{C}$,

\footnotetext{
${ }^{1}$ To check whether $\mathcal{E}$ satisfies the conditions, substitute $\mathcal{E}$ for $\mathcal{C}$ in Lem. 1 .
}

which is equivalent to $\left\{x \mid\|x\|_{Q}^{2} \leq 1\right\} \subseteq\left\{x \mid H x \leq \rho_{1} \mathbf{1}\right\}$. Using the coordinate transformation $x=L^{-T} \xi$, this can be rewritten as $\left\{\xi \mid\|\xi\|_{2}^{2} \leq 1\right\} \subseteq\left\{\xi \mid H L^{-T} \xi \leq \rho_{1} \mathbf{1}\right\}$, which immediately leads to (29). Now, the relation

$$
\mu=\frac{1}{\rho_{1}^{2}} \leq l^{\star}(x) \text { for every } \quad x \in \mathcal{X} \backslash \operatorname{int}(\mathcal{C}),
$$

can be proven analogously to relation (24) in Prop. 2. It remains to show

$$
V_{\infty}(x) \leq \frac{\max \left\{\rho_{1} \rho_{2}, \rho_{2}^{2}\right\}}{\eta} l^{\star}(x) \text { for every } \quad x \in \mathcal{C} .
$$

Based on (31) and the assumptions on $\mathcal{C}$ and $\mathcal{E}$, we have $V_{\infty}(x) \leq \frac{1}{\eta} \Psi_{\mathcal{E}}^{2}(x) \leq \frac{\rho_{2}^{2}}{\eta} \Psi_{\mathcal{L}}^{2}(x)=\frac{\rho_{2}^{2}}{\eta} l^{\star}(x)$ for every $x \in \mathcal{E}$, which proves (33) for $x \in \mathcal{E}$, and

$$
V_{\infty}(x) \leq \frac{1}{\eta} \Psi_{\mathcal{C}}(x) \leq \frac{\rho_{1}}{\eta} \Psi_{\mathcal{L}}(x) \quad \text { for every } \quad x \in \mathcal{C} .
$$

Consider any $x \in \mathcal{C} \backslash \mathcal{E}$. Then, we have $\Psi_{\mathcal{E}}(x)>1$ and consequently $\rho_{2} \Psi_{\mathcal{L}}(x) \geq \Psi_{\mathcal{E}}(x)>1$. Thus, we obtain

$$
V_{\infty}(x) \leq \frac{\rho_{1}}{\eta} \Psi_{\mathcal{L}}(x)<\frac{\rho_{1} \rho_{2}}{\eta} \Psi_{\mathcal{L}}^{2}(x) \quad \text { for every } \quad x \in \mathcal{C} \backslash \mathcal{E},
$$

from (34), which completes the proof.

Remark 4: Obviously, Prop. 3 is useful only if $\rho_{1} \leq \rho_{2}$. Otherwise, we gain $\gamma=\frac{\rho_{1} \rho_{2}}{\eta}>\frac{\rho_{2}^{2}}{\eta}$ and $\mu=\frac{1}{\rho_{1}^{2}}<\frac{1}{\rho_{2}^{2}}$ and we obtain better results by directly applying Cor. 3 to the auxiliary ellipsoidal set $\mathcal{E}$. If $\rho_{1} \leq \rho_{2}$, we obtain $\gamma=\frac{\rho_{2}^{2}}{\eta}$ and $\mu=\frac{1}{\rho_{1}^{2}} \geq \frac{1}{\rho_{2}^{2}}$ and the consideration of the polytope (in terms of $\rho_{1}$ ) may lead to a smaller value $\mu$. Let $\rho_{1}^{*}$ and $\rho_{2}^{*}$ be the smallest values satisfying (29) and (30), respectively. Then, we obviously have $\rho_{1}^{*} \leq \rho_{2}^{*}$ if (and only if) $\mathcal{E} \subset \mathcal{C}$.

\section{NUMERICAL EXAMPLE}

In the previous section, we showed that terminal sets and costs originally designed for MPC schemes with terminal constraints can also be used to tune stabilizing MPC schemes without terminal constraints. In this section, we compare the resulting controllers by analyzing an example. We consider a discrete-time variant ${ }^{2}$ of the bilinear system introduced in [2] (and also analyzed in [7], [8], [10]). The system is given by

$$
f(x, u)=\left(\begin{array}{l}
x_{1}+0.1 x_{2}+0.09 u+0.01 x_{1} u \\
x_{2}+0.1 x_{1}+0.09 u-0.04 x_{2} u
\end{array}\right)
$$

with the state and input constraints

$$
\mathcal{X}=\left\{x \in \mathbb{R}^{2} \mid\|x\|_{\infty} \leq 4\right\} \quad \text { and } \mathcal{U}=[-2,2] .
$$

Analogously to $[2]^{3}$, we consider the quadratic stage (14) with $Q=\left(\begin{array}{ll}0.05 & 0.00 \\ 0.00 & 0.05\end{array}\right)$ and $R=0.1$. To apply the criteria in Sect. III-A, first note that (35) can be easily bounded by LDIs as discussed in [8, Sect. 2]. In particular, for any polytopic C-set $\mathcal{C}$ with $\left\{v_{1}, \ldots, v_{r}\right\}=\operatorname{extr}(\mathcal{C})$, the choice

$$
A_{i}=\left(\begin{array}{ll}
1.0 & 0.1 \\
0.1 & 1.0
\end{array}\right) \text { and } B_{i}=\left(\begin{array}{l}
0.09 \\
0.09
\end{array}\right)+\left(\begin{array}{rr}
0.01 & 0.00 \\
0.00 & -0.04
\end{array}\right) v_{i}
$$

${ }^{2}$ Euler discretization of [2, Eq. (36)] with $\Delta t=0.1$ and $\mu=0.9$.

${ }^{3}$ We consider $Q$ and $R$ from [2, Eq. (38)] multiplied with $\Delta t=0.1$. 
is such that (13) holds. Based on this observation, it is straightforward to verify ${ }^{4}$ that the sets $\mathcal{E}=\left\{x \mid x^{T} P x \leq 1\right\}$ and $\mathcal{C}=\left\{x \in \mathbb{R}^{n} \mid H x \leq \mathbf{1}\right\}$ with

$P=\left(\begin{array}{ll}0.5382 & 0.0000 \\ 0.0000 & 0.5382\end{array}\right)$ and $H=\left(\begin{array}{rr}0.7064 & 0.6543 \\ -0.1358 & 0.5032 \\ -0.7064 & -0.6543 \\ 0.1358 & -0.5032\end{array}\right)$

satisfy the conditions in Cors. 1 and 2 for $\eta=0.05$, respectively, and thus can both be used as terminal sets in stabilizing MPC with terminal constraints. Since

$$
\operatorname{vol}(\mathcal{C})=9.0016>5.8375=\operatorname{vol}(\mathcal{E}),
$$

the polytopic set $\mathcal{C}$ is a better choice for the terminal set $\mathcal{T}$ in (2) since it promotes a larger feasible set and thus a larger DOA (see Thm. 1). The resulting DOA $\mathcal{D}_{N}^{\text {Thm. }}{ }^{1}$ is illustrated in Fig. 1 for different prediction horizons $N \in$ $\{2,5,10,20,50\}$.

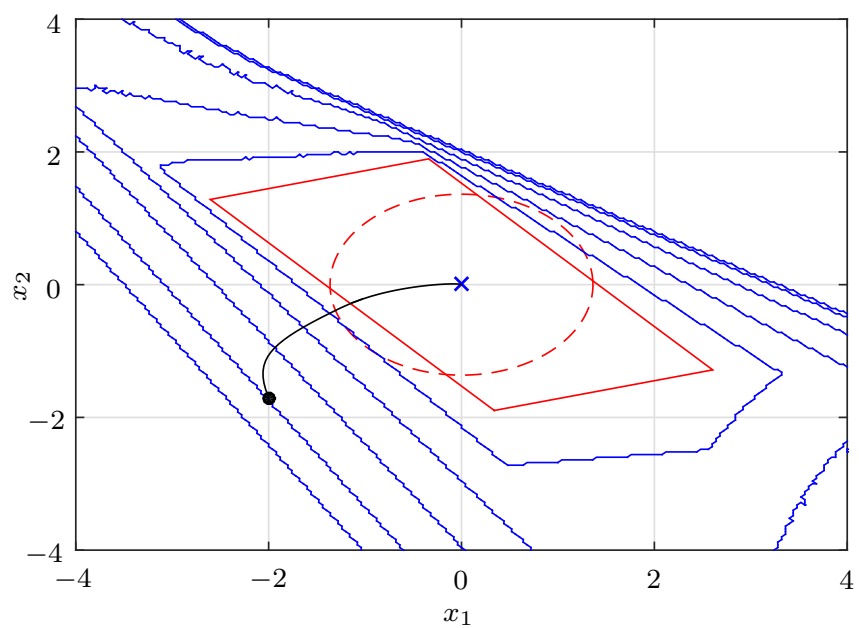

Fig. 1. Approximation of the DOA $\mathcal{D}_{N}^{\text {Thm. } 1}$ for the MPC scheme with terminal constraints $(\mathcal{T}=\mathcal{C})$ and different predictions horizons $N$. The blue framed sets refer (from inside to outside) to the DOA for $N$ equal to $2,5,10,20$, and 50 , respectively. The red solid polytope illustrates the set $\mathcal{C}$. The red dashed ellipsoid depicts $\mathcal{E}$ for ease of comparison. The black trajectory refers to a closed-loop trajectory resulting for the MPC law $\varrho_{50}^{\text {Thm. } 1}(x)$ from (3), respectively.

To prepare the re-utilization of the sets for MPC without terminal constraints, we first compute the smallest $\rho$ satisfying (26) and obtain $\rho^{*}=3.2823$. Note that the application of Prop. 3 is not reasonable since $\mathcal{E} \not \subset \mathcal{C}$ (see Fig. 2 and Rem. 4). Computing $\gamma$ and $\mu$ according to Cor. 3 leads to $\gamma=122.135$ and $\mu=0.093$. Choosing $\delta=20$ implies $\beta=\gamma=\frac{\delta}{\mu}$. Finally, the smallest $N$ satisfying (10) reads $N=1154$. Thus, according to Thm. 2, the MPC (2) with $\mathcal{T}=\mathcal{X}, \tau(x)=l^{\star}(x)=\|x\|_{Q}^{2}$, and $N=1154$ will stabilize every $x_{0} \in \mathcal{X}$ with $V_{1154}^{\text {Thm }}{ }^{2}\left(x_{0}\right) \leq 20$. In Fig. 2 the set $\left\{x_{0} \in \mathcal{X} \mid V_{100}^{\text {Thm. } 2}\left(x_{0}\right) \leq \delta\right\}$ is illustrated for different values $\delta \in\{1,2,5,10,20\}$.

Obviously, the size (and shape) of the set $\left\{x_{0} \in\right.$ $\left.\mathcal{X} \mid V_{100}^{\text {Thm. }}{ }^{2}\left(x_{0}\right) \leq 20\right\}$ is comparable to the DOA obtained

\footnotetext{
${ }^{4}$ We have considered the axis-aligned bounding of $\mathcal{E}$ to compute an LDI
} for $(35)$ on $\mathcal{E}$. for $N=50$ in Fig. 1. Since

$$
\left\{x_{0} \in \mathcal{X} \mid V_{N}^{\text {Thm. } 2}\left(x_{0}\right) \leq 20\right\} \subseteq\left\{x_{0} \in \mathcal{X} \mid V_{100}^{\text {Thm. }}{ }^{2}\left(x_{0}\right) \leq 20\right\}
$$

for every $N \geq 100$, this observation implies that we obviously need to consider a $\delta \geq 20$ in order to obtain a guaranteed DOA that is comparable to $\mathcal{D}_{50}^{\text {Thm. } 1}$ from Fig. 1 . At this point, it is important to note that the set $\mathcal{E}$ was designed in such a way that $\beta$ and consequently $N$ become as small as possible ${ }^{5}$ for the specification $\delta=20$. In other words, it is hard to find $P, K, \eta$, and $\rho$ satisfying the conditions in Cors. 1 and 3 (using LDIs as in footnote 4) such that the 1.h.s. in (10) becomes significantly smaller than 1154 for $\beta=\max \left\{\delta \rho^{2}, \frac{\rho^{2}}{\eta}, 2\right\}$. Clearly, we are able to realize smaller prediction horizons $N$ if we consider smaller $\delta \in \mathbb{R}_{+}$, i.e., smaller guaranteed DOA (see Fig. 2). However, even for the choice $\delta=1$, we require $N=171$ according to Tab. II. At this point, it is important to note that $\mathcal{D}_{N}^{\mathrm{Thm} .} 2$ only represents an inner approximation of the actual DOA. In fact, the actual DOA may be relatively large even for relatively small $N$. However, there is in general no systematic way to compute the actual DOA.

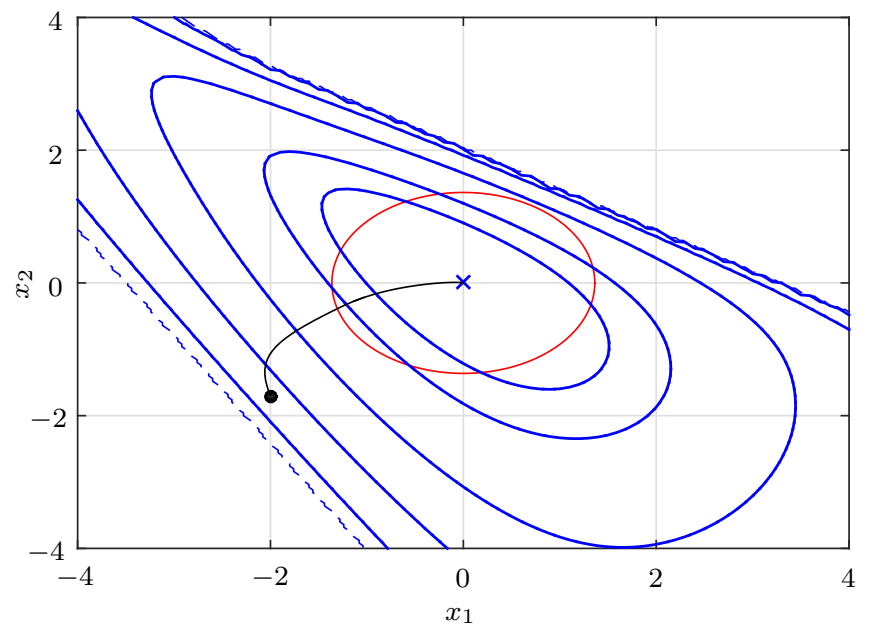

Fig. 2. Approximation of the set $\mathcal{D}_{100}^{\text {Thm. }}{ }^{2}=\left\{x_{0} \in \mathcal{X} \mid V_{100}^{\text {Thm. }}{ }^{2}\left(x_{0}\right) \leq \delta\right\}$ for the MPC scheme without terminal constraints $(\mathcal{T}=\mathcal{X})$ and different values $\delta$. The blue framed sets refer (from inside to outside) to $\mathcal{D}_{100}^{\mathrm{Thm} .} 2$ with $\delta$ equal to $1,2,5,10$, and 20 , respectively. The red solid ellipsoid illustrates $\mathcal{E}$. The blue dashed set shows $\mathcal{D}_{50}^{\text {Thm. } 1}$ from Fig. 1 for ease of comparison. The black trajectory refers to a closed-loop trajectory resulting for the MPC law $\varrho_{100}^{\text {Thm. }} 2(x)$ from (3).

Comparison of the DOA in Figs. 1 and 2 shows that, for this example and using the methods introduced in Sect. IV, we require significantly longer prediction horizons for the MPC without terminal constrait to archive a guaranteed DOA that is comparable to the DOA of the MPC with terminal constraints and $N=50$. This observation was anticipated in the introduction and states an advantage of the MPC scheme with terminal constraints. It is, however, important to keep in mind that the relation

$$
V_{N}^{\text {Thm. } 2}\left(x_{0}\right) \leq V_{\infty}\left(x_{0}\right) \leq V_{M}^{\text {Thm. }}\left(x_{0}\right)
$$

holds for arbitrary $N, M \in \mathbb{N}_{+}$.

\footnotetext{
${ }^{5}$ In contrast to $\mathcal{E}$, the set $\mathcal{C}$ was designed to provide an as large as possible volume (see [8] for details).
} 
TABLE II

Approximations of the smallest prediction horizon $N$ satisfying (10) for different values $\delta$ based on $P, K, \eta, \rho, \gamma$, and $\mu$ as in Cors. 1 and 3 .

\begin{tabular}{rrrrrr}
\hline & \multicolumn{5}{c}{$\delta$} \\
\cline { 2 - 6 } & \multicolumn{1}{c}{1} & \multicolumn{1}{c}{2} & \multicolumn{1}{c}{5} & \multicolumn{1}{c}{10} & \multicolumn{1}{c}{20} \\
\hline$\eta$ & 1.000 & 0.500 & 0.200 & 0.100 & 0.050 \\
$\gamma$ & 45.238 & 52.729 & 76.496 & 122.135 & 215.269 \\
$\mu$ & 0.022 & 0.038 & 0.065 & 0.082 & 0.093 \\
\hline$N$ & 171 & 208 & 330 & 585 & 1154 \\
\hline
\end{tabular}

Clearly, the overestimation $V_{M}^{\text {Thm. } 1}(x) \geq V_{\infty}(x)$ is a direct consequence of the terminal set and cost. In fact, especially if $\eta$ is chosen small in order to obtain a large terminal set, the terminal cost $\varphi(x)=\frac{1}{\eta} \Psi_{\mathcal{C}}(x)$ overestimates $V_{\infty}(x)$ significantly. This becomes apparent if we consider a state $x_{0} \in \mathcal{X}$ and a horizon $N$ for which (2) is feasible using $\mathcal{T}=\mathcal{C}$, but where the optimal $x^{*}(N)$ lies in the boundary area of the terminal set $\mathcal{C}$. Regarding Fig. 1, this case occurs for $x_{0}=\left(\begin{array}{ll}-2.0 & -1.7\end{array}\right)^{T}$ and $N=20$. Evaluating the cost function value $V_{20}^{\text {Thm. }}{ }^{1}(x(k))$ along the closed-loop trajectory leads to the trend in Fig. 3. Compared to the evolution of $V_{100}^{\text {Thm. }} 2(x(k))$ and $V_{50}^{\text {Thm. }}{ }^{1}(x(k)), V_{20}^{\text {Thm. } 1}(x(k))$ shows a significant overshoot especially for the first five steps. Since $V_{100}^{\text {Thm. }}{ }^{2}(x(k))$ and $V_{50}^{\text {Thm. }}{ }^{1}(x(k))$ are almost identical, i.e., $V_{100}^{\text {Thm. } 2}(x(k)) \approx V_{50}^{\text {Thm. } 1}(x(k)) \approx V_{\infty}(x(k))$ according to $(36), V_{20}^{\text {Thm. } 1}(x(k))$ represents a bad approximation of the original performance measure. Nevertheless, the closed-loop trajectory is visually indistinguishable from the trajectories illustrated in Figs. 1 and 3.

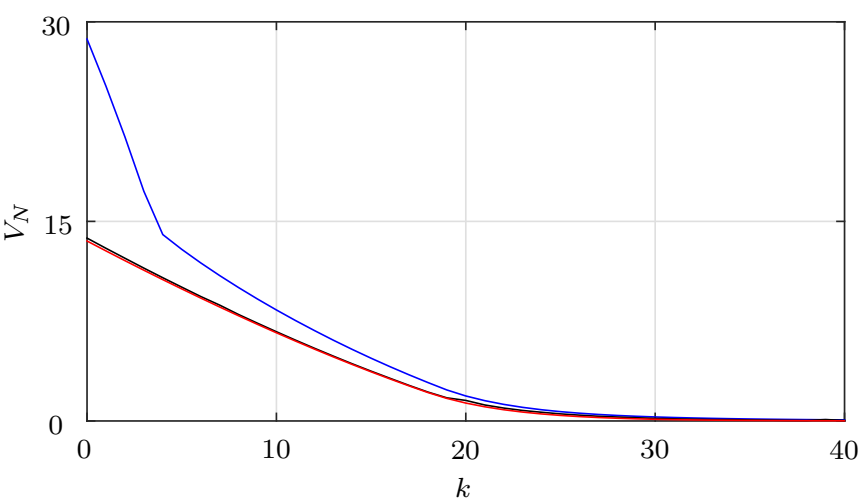

Fig. 3. Evolution of $V_{100}^{\text {Thm. }} 2(x(k))$ (red), $V_{50}^{\text {Thm. } 1}(x(k))$ (black), and $V_{20}^{\text {Thm. }}(x(k))$ (blue) along the respective closed-loop trajectory.

\section{CONCLUSION AND OUTLOOK}

The paper presents a missing link between stabilizing MPC schemes with and without terminal constraints (see Thms. 1 and 2 or [1]-[5]). Basically, we show that terminal sets and costs designed for MPC with terminal constraints can be exploited to compute suitable prediction horizons for stabilizing MPC without terminal constraints. In particular, we provide a method to systematically verify the central condition (10) on $N$ as introduced in [4]. The main result of the paper is summarized in Prop. 2. Corollaries 3 through 5 and Prop. 3 state customized variants for different sets (ellipsoidal or polytopic) and costs (quadratic or linear).

The presented results were illustrated with a numerical example. The example shows that the computation of prediction horizons satisfying condition (10) can easily be carried out based on the knowledge of terminal sets (and costs) using the proposed method. However, the computed horizons are large ( $N \in \mathbb{N}_{[171,1154]}$ for the discussed example) and may not be suitable for a practical realization of the MPC.

We have to stress that this observation does not imply that stabilizing MPC without terminal constraints is not useful in practice. In fact, a number of well-functioning examples are presented in [4], [5]. Basically, the motivation for this paper was to illustrate the link between the discussed stabilizing MPC schemes and to simultaneously provide a method to systematically verify condition (10) for a huge class of systems. The analyzed example simply illustrates that this link is currently not "strong" enough to provide efficient verification procedures.

Nevertheless, we think that the presented link is interesting and may be further exploited. Consequently, future work has to address the improvement of the link from two directions. First, the conservatism inherent in (6) can be reduced using sharper criteria to identify appropriate terminal sets and costs. Second, it may be possible to improve criterion (10) for the suitable choice of the prediction horizon $N$. Note that an improvement of the presented condition (22) is impossible since it is necessary and sufficient according to Lem. 1.

\section{REFERENCES}

[1] D. Q. Mayne, J. B. Rawlings, C. Rao, and P. O. M. Scokaert, "Constrained model predictive control: Stability and optimality," $A u$ tomatica, vol. 36, pp. 789-814, 2000.

[2] H. Chen and F. Allgöwer, "A quasi-infinite horizon nonlinear model predictive control scheme with guaranteed stability," Automatica, vol. 34, no. 10, pp. 1205-1217, 1998.

[3] G. De Nicolao, L. Magni, and R. Scattolini, "Stabilizing nonlinear receding horizon control via a nonquadratic penalty," in Proceedings of the IMACS multiconference CESA, pp. 185-187, 1996.

[4] A. Boccia, L. Grüne, and K. Worthmann, "Stability and feasibility of state constrained MPC without stabilizing terminal constraints," System and Control Letters, vol. 72, pp. 14-21, 2014.

[5] L. Grüne, "Analysis and design of unconstrained nonlinear MPC schemes for finite and infinite dimensional systems," SIAM Journal on Control and Optimization, vol. 48, no. 2, pp. 1206-1228, 2009.

[6] A. Jadbabaie and J. Hauser, "On the stability of receding horizon control with a general terminal cost," IEEE Trans. Autom. Control, vol. 50, no. 5, pp. 674-678, 2005.

[7] W.-H. Chen, D. J. Ballance, and J. O'Reilly, "Optimisation of attraction domains of nonlinear MPC via LMI methods," in Proc. of the 2001 American Control Conference, pp. 3067-3072, 2001.

[8] M. Cannon, V. Deshmukh, and B. Kouvaritakis, "Nonlinear model predictive control with polytopic invariant sets," Automatica, vol. 39, pp. 1487-1494, 2003

[9] T. B. Blanco, M. Cannon, and B. De Moor, "On efficient computation of low-complexity controlled invariant sets for uncertain linear systems," International Journal of Control, vol. 83, no. 7, pp. 13391346, 2010.

[10] M. Fiacchini, T. Alamo, and E. F. Camacho, "On the computation of convex robust control invariant sets for nonlinear systems," Automatica, vol. 46, pp. 1334-1338, 2010.

[11] F. Blanchini and S. Miani, Set-Theoretic Methods in Control. Birkhäuser, 2008. 